\title{
CONTROL PREEMERGENTE Y POSTEMERGENTE DE MALEZAS EN FRIJOL, DE HUMEDAD RESIDUAL EN VERACRUZ, MEXICO ${ }^{1}$
}

\author{
Octavio Cano ${ }^{2}$, Ernesto López ${ }^{2}$
}

\section{RESUMEN}

Control preemergente y postemergente de malezas en frijol, de humedad residual en Veracruz, México. En el ciclo Otoño Invierno 1993 - 1994, se realizó en el Campo Experimental Cotaxtla un estudio con el objetivo de determinar los herbicidas pre y postemergentes más eficientes para controlar malezas en forma óptima y económica en frijol. Las malezas dominantes en el sitio experimental fueron, flor amarilla (Aldama dentata) y zacate pitillo (Ixoporus unisetus). Los herbicidas preemergentes Metribuzina 0,350 kg i.a./ ha, Alaclor + Metribuzina 1,5+0,210 y 1,5+0,290 kg i.a/ha , controlaron zacates y hoja ancha en forma aceptable, el tratamiento óptimo económico fue Alaclor + Metribuzina 1,5 + $0,210 \mathrm{~kg}$ i.a./ha con una TMR de $1655 \%$ y RBC de 10,6 . El mejor herbicida postemergente para controlar malezas de hoja ancha y zacates, fue la mezcla de Fluazifop Butil + Fomesafén $0,125+0,25 \mathrm{~kg}$ i.a./ha siendo el mejor postemergente óptimo económico con una TMR de $36 \%$ y RBC de 6,3; obteniendo el máximo rendimiento $1061 \mathrm{Kg} / \mathrm{ha}$. Imazethapyr y Chlorimuron Ethyl resultaron altamente tóxicos para el frijol (100\%).

\begin{abstract}
Pre-emergent and post-emergent control of weeds in residual humidity beans, in Veracruz, México. In the period from the Fall of 1993 to the Winter of 1994, at the Cotaxtla Experimental Field, a research with the goal of determining the most efficient pre-emergent and post-emergent herbicides was performed, in order to control weeds in bean plantations in the optimal and most economic way. The dominant weeds at the experimental site were: Yellow Flower (Aldama dentata) and "pitillo" grass (Ixoporus unisetus). The pre-emergent herbicides: Metribuzina, $0.350 \mathrm{Kg}$ i.a./Ha, Alaclor + Metribuzina $1.5+0.210$ and $1.5+0.290 \mathrm{Kg}$ i.a. $/ \mathrm{Ha}$, were found to control grass and broad leaves in an acceptable way. The optimal treatment was that of Alaclor + Metribuzina $1.5+0.210 \mathrm{~kg}$ i.a./ha with a TMR of $1655 \%$ and an RBC of 10.6 . The best post-emergent herbicide to control broad-leaf weeds and grass, was the mixture of Fluazifop Butil + Fomesafen $0.125+0.25 \mathrm{~kg}$ i.a./ha, as the most economic and optimal post-emergent with a TMR of $36 \%$ and an RBC of 6.3 . We obtained the best yields with $1061 \mathrm{~kg} / \mathrm{ha}$. Imazethapyri Chlorimuron Ethyl were found to be highly toxic for the bean plant (100\%).
\end{abstract}

\section{INTRODUCCION}

En 1992, en el estado de Veracruz se sembraron 33,922 hectáreas de frijol que produjeron más de 23,000 toneladas, con un promedio en rendimiento de $678 \mathrm{~kg} / \mathrm{ha}$.

Los bajos rendimientos se deben a un gran número de factores limitantes entre los que destaca la maleza. En un estudio de factibilidad para la producción artesanal de semilla en la región de la Mixtequilla, Ver., se encontró que el $72 \%$ de los productores de la zona controla malezas en forma manual, con tarpala o azadón, práctica que requiere excesiva mano de obra y es de reducida eficiencia, ya que las malezas que se desarrollan en la hilera del surco son difíciles de combatir, ocasionando una disminución en el rendimiento mayor al 50\%, además de afectar la calidad de grano.

La problemática anterior llevó a la búsqueda de mejores opciones en el control de maleza, siendo el control químico una buena alternativa.

Con base en lo anteriormente expuesto, se realizó el presente estudio con el objetivo de determinar los

1 Presentado en la XLI Reunión Anual del PCCMCA en Honduras, América Central. 26 de marzo - 1 de abril, 1995.

2 Programa de Leguminosas Comestibles del Campo Experimental Cotaxtla. INIFAP. Apdo. Postal 429. Veracruz, México. 
herbicidas preemergentes y postemergentes más eficientes para controlar malezas en frijol, bajo condiciones de humedad residual, en forma óptima económica.

En un estudio realizado en Tuxpan, Nayarit por Ríos y Pérez (1990) para determinar el mejor tratamiento para el control de maleza en postemergencia en frijol, se evaluaron los herbicidas Fomesafén 1,0 y 1,5 1/ha, Imazethapyr 0,75 y 1 l/ha, Acifluorfen 1,0 y 1,5 1/ha y Bentazón 2 1/ha, encontraron como malezas dominantes quelites (Amaranthus spp), Alderete (Xanthium pensylvanicum), coquillo (Cyperus spp), Gloria de la mañana (Ipomea spp), zacate pinto (Echinochloa cruzgalle) y mancamula (Solanum rostratum) ; los herbicidas presentaron controles superiores al $90 \%$, excepto acifluorfen con control de $80 \%$, la toxicidad más alta fue para acifluorfen con $30 \%$ seguida de Imazethapyr con 10\%. Los mayores rendimientos se obtuvieron con fomesafén 2,0 1/ha siendo $2239 \mathrm{~kg} / \mathrm{ha}$.

Considerando el control de maleza, la fitoxicidad y rendimiento al evaluar la actividad biológica de diversos herbicidas en frijol ejotero, Bolaños et al. (1993) obtuvo el mejor tratamiento con Fomesafén en postemergencia en dosis $0,25 \mathrm{~kg}$ i.a./ha, mientras que Acifluorfen y Bentazón mostraron un alto grado de toxicidad al cultivo.

Castro (1993) encontró que los herbicidas Fomesafén en 0,375 y $0,750 \mathrm{~kg}$ i.a./ha, Imazethapyr $0,1 \mathrm{y}$ $0,15 \mathrm{~kg}$ i.a./ha controlaron eficazmente el cadillo (Xanthium strumarium L.). Además reportó que los herbicidas Fomesafén y Bentazón fueron altamente, selectivos a frijol mientras que Imazethapyr causo clorosis ligera 6-8\% que desapareció a los treinta y cinco días después de aplicado el producto; mientras que el 2,4-DB fue altamente tóxico al grado de reducir la producción en un $40 \%$.

Tamayo (1991), indica que los herbicidas más adecuados para control de maleza en frijol son Alaclor, Metabenstiazuron, Linuron, Clorobromuron, Pendimetalina, Dinoseb, Fomesafén y Fluazifop Butil; además que con mezclas de algunos de estos productos se incrementa la eficiencia de control.

Cano y López (1993) afirman que Fomesafén tiene una alta eficiencia en el control de maleza de hoja ancha y una alta selectividad para el frijol; por el contrario que Ríos y Rojas (1987) indican que debido a los efectos fitotoxicos del Fomesafén en frijol, no se deben aplicar dosis mayores a $0,6 \mathrm{~kg}$ i.a./ha en plantas con tres hojas trifoliadas o menos, para evitar atraso en el desarrollo del cultivo.
Cid y Torres (1987) indican que Fomesafén en dosis de 1,5 1/ha controla mas eficientemente la maleza 30 días después de aplicado, comparado con Bentazón. En este sentido Morgado y Tafoya (1990), señalan que la aplicación de Fomesafén en preemergencia al cultivo y a la maleza en dosis de $0,375 \mathrm{~kg}$ i.a./ha y la mezcla de este con $0,375 \mathrm{~kg}$ i.a./ha de Fluazifop Butil en postemergencia se obtienen controles superiores a $80 \%$. Al respecto, Ramírez y Tafoya (1990), encontraron que agronomicamente el mejor tratamiento para controlar la maleza en postemergencia y preemergencia fue Fomesafén 0,375 y $0,500 \mathrm{~kg}$ i.a. $/ \mathrm{ha}$ respectivamente.

Esqueda, López y Cano (1992) afirman que Fomesafén en dosis de $0,187 \mathrm{~kg}$ i.a./ha controla eficientemente maleza de hoja ancha hasta de $8 \mathrm{~cm}$ de altura, mientras que cuando la maleza tiene entre 10 y 60 $\mathrm{cm}$ es necesario aplicar $0,375 \mathrm{~kg}$ i.a./ha. Los mismos autores encontraron control de maleza eficiente con Fomesafén en dosis de 0,250 y 0,375 kg i.a./ha aplicado en postemergencia, señalan que Linuron y la mezcla de Alaclor+Linuron mostraron controles regulares a buenos en preemergencia pero no comparables a los obtenidos con Fomesafén.

\section{MATERIALES Y METODOS}

El experimento se estableció en el ciclo otoñoinvierno 1993 - 1994, en el lote D5 del Campo Experimental Cotaxtla del Instituto Nacional de Investigaciones Forestales y Agropecuarias (INIFAP), localizado en el Km 34,5 de la Carretera Veracruz Córdoba, en el Municipio de Medellín de Bravo, Ver. La fecha de siembra fue el siete de octubre de 1993.

La siembra se realizó con sembradora mecánica (MP25), en surcos separados a $60 \mathrm{~cm}$, depositando de 15 a 18 semillas por metro lineal de surco, para ajustar una densidad de población de 250,000 plantas por hectárea. Se sembró la variedad Negro Cotaxtla-91 y se fertilizó a la siembra con la fórmula 40-40-00 de $\mathrm{N}_{2} \mathrm{P}_{2} \mathrm{O}_{5}$ y $\mathrm{K}_{2} 0$ respectivamente. Se utilizó un diseño experimental en bloques al azar con 16 tratamientos y cuatro repeticiones (Cuadro 1).

La parcela experimental constó de cuatro surcos de seis metros de longitud. Como parcela útil se tomaron los dos surcos centrales, eliminando 0,5 metros en los extremos. Los tratamientos se aplicaron con bomba de mochila con motor a gasolina, usando boquillas de abanico Tee -Jeet 8002, con un gasto de agua de 365,2 1/ha. Los herbicidas preemergentes y postemergentes se 
Cuadro 1. Tratamientos de evaluación de herbicidas pre y postemergentes evaluados en frijol de humedad residual, en el Centro de Veracruz. Ciclo Otoño-Invierno 1993-1994.

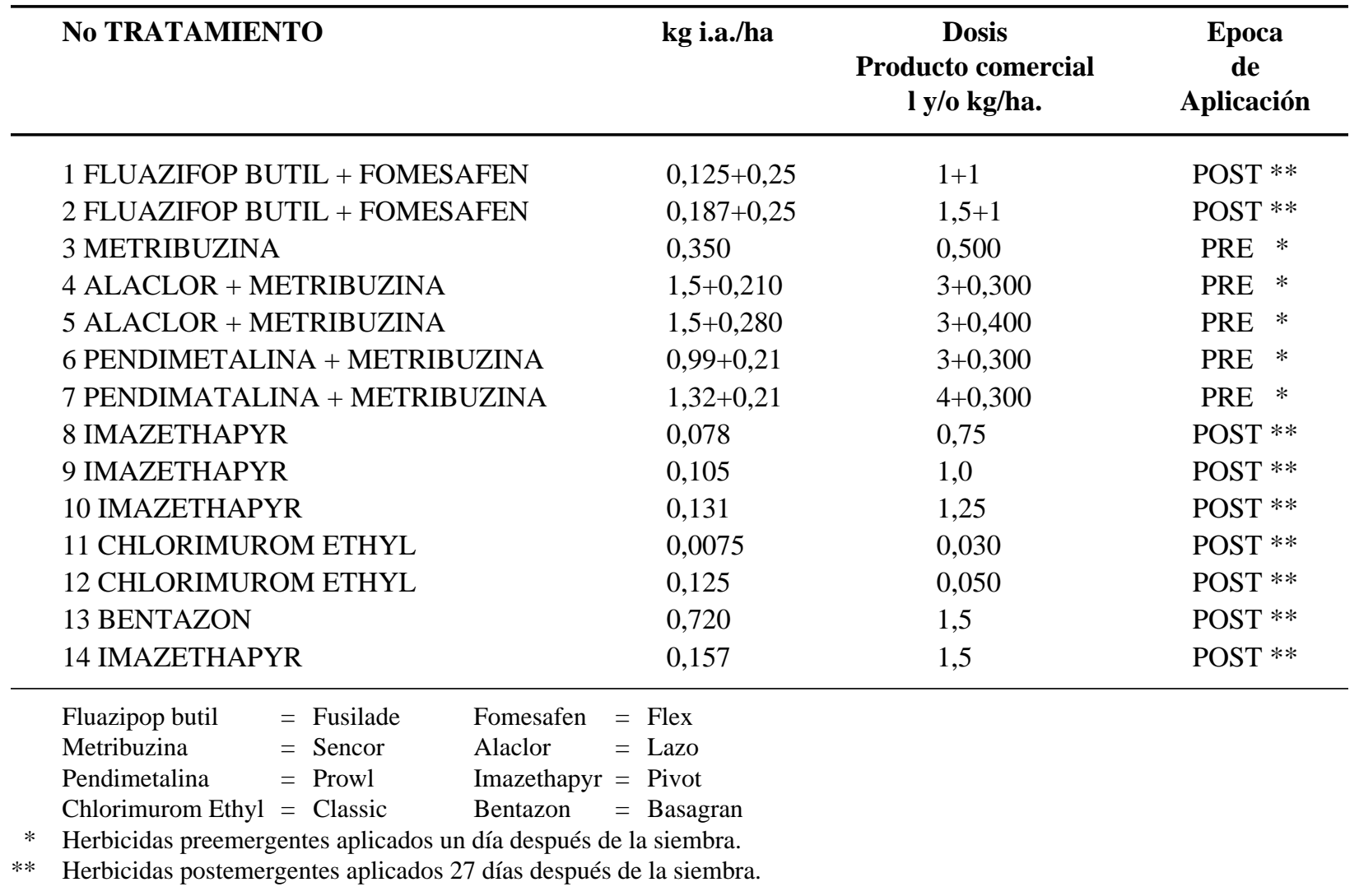

aplicaron el día 8 de octubre y 11 de noviembre de 1993 respectivamente.

Se identificaron las malezas presentes en el sitio experimental y se determinó su densidad de población (DDP), a los 33 y 42 días después de la emergencia del cultivo (DDE), con un cuadrante de $0,50 \mathrm{~m} \mathrm{X} 0,50 \mathrm{~m}$ lanzado en forma aleatoria sobre los testigos enhierbados.

Para evaluar la eficiencia de los tratamientos, se estimó el porcentaje de control y toxicidad al cultivo de los herbicidas a los 15 y 30 días después de aplicados (DDA), usando una escala visual de 0 a $100 \%$, donde cero indica que no hubo control o toxicidad, y 100 que controló o hubo toxicidad en forma total.

Además se midieron las variables altura de planta a madurez fisiológica, plantas cosechadas (miles/ha) y rendimiento de grano en $\mathrm{kg} / \mathrm{ha}$ al $14 \%$ de humedad. Se determinó la altura final de maleza como dato comparativo con el crecimiento del cultivo.
Se realizó análisis de varianza para las variables estudiadas y prueba de Tukey al 0,05 de probabilidad, para la separación de tratamientos. Con el rendimiento se determinó el tratamiento óptimo económico, utilizando el análisis de la tasa marginal de retorno (TMR) y la relación beneficio costo (RBC).

El manejo del cultivo fue el recomendado en el paquete tecnológico para frijol del Campo Experimental Cotaxtla (CECOT), dependiente del Insituto Nacional de Investigaciones Forestales y Agropecuarias (INIFAP).

\section{RESULTADOS Y DISCUSION}

Los resultados sobre las malezas presentes en el sitio experimental se señalan en el (Cuadro 2); se identificaron ocho especies de maleza pertenecientes a siete familias botánicas: 2 Poaceae, 1 Asteraceae, 1 Malvaceae, 1 Euphorbiaceae, 1 Portulacaceae y 1 Papaveraceae. 
Cuadro 2. Malezas presentes en el sitio experimental. Ciclo Otoño-Invierno 1993-1994. CECOT. CIRGOC. INIFAP. SARH.

\begin{tabular}{|c|c|c|}
\hline Nombre Común & Nombre Científico & Familia \\
\hline Flor amarilla La llave \& lex & Aldama dentata & Asteraceae \\
\hline Zacate pitillo (Presl) Schuhlt & Ixophorus unisetus & Poaceae \\
\hline Malva peluda Jacq. & Malachra fasciata & Malvaceae \\
\hline Zacate de agua L./Link. & Echinochloa colonum & Poaceae \\
\hline Coquillo & Cyperus rotundus L. & Cyperaceae \\
\hline Collarcillo & Phyllanthus niruri L. & Euphorbiaceae \\
\hline Verdolaga & Portulaca oleracea & Portulacaceae \\
\hline Carlo santo & Argemone ochroleuca $\mathrm{L}$. & Papaveraceae \\
\hline Leche de sapo & Euphorbia heteropylla & Euphorbiaceae \\
\hline
\end{tabular}

La densidad de población de malezas a los 33 y 42 días después de emergido el cultivo fue de $1.620,000$ y 2'130,000 plantas/ha respectivamente, considerándose alta con respecto a la densidad de población del cultivo. La primera evaluación de densidad de población de malezas se realizó hasta los 33 días después de emergido el cultivo; debido a la falta de humedad los primeros 15 días después de nacido el cultivo que ocasionó que no emergieran malezas, por lo que fue necesario regar para inducir la germinación de malas hierbas (Cuadro 3).

Como especies dominantes en la primera evaluación 33 (DDE) se presentó la flor amarilla (Aldama dentata) y el zacate pitillo (Ixoporus unisetus) con 47,53 y $19,75 \%$ respectivamente; en la segunda evaluación predominaron las mismas con 42,25 y $17,84 \%$, respectivamente; porcentaje de dominancia alto, dado que entre estas dos especies superan más del $60 \%$ de la densidad de población total de maleza. Además de las malezas dominantes en la primera y segunda evaluación, se presentó la malva peluda (Malachra fasciata), zacate de agua (Echinochloa colona), coquillo (Cyperus rotundus), verdolaga (Portulaca oleracea), collarcillo (Phyllanthus niruri) y cardo santo (Argemone achroleuca) con valores inferiores a 9,88 \%; la variación de especies de malezas nos permitió observar qué tan efectivos fueron los herbicidas para controlar malezas, (Cuadro 3).

El control de malezas de hoja ancha, zacates y cyperaceas se presenta en el Cuadro 4; Metribuzina en dosis de $0,350 \mathrm{~kg}$ i.a./ha controló en forma regular hoja

Cuadro 3. Densidad de población de malezas a los 33 y 42 días después de emergido el cultivo de frijol (dde). Ciclo Otoño-Invierno 1993-1994. CECOT. CIRGOC. INIFAP. SARH.

\begin{tabular}{lcccc}
\hline \multirow{2}{*}{\begin{tabular}{l} 
Combre \\
\cline { 2 - 5 }
\end{tabular}} & $\mathbf{3 3}^{*}$ & \% Relativo & $\mathbf{4 2 *}$ & \% Relativo \\
\hline Flor amarilla & 770 & 47,53 & 900 & 42,25 \\
Zacate pitillo & 320 & 19,75 & 380 & 17,84 \\
Malva peluda & 160 & 9,88 & 200 & 9,39 \\
Zacate de agua & 150 & 9,25 & 170 & 7,98 \\
Coquillo & 130 & 8,02 & 190 & 8,92 \\
Collarcillo & 50 & 3,08 & 130 & 6,10 \\
Verdolaga & 20 & 1,23 & 50 & 2,35 \\
Carlo santo (Chicalote) & 20 & 1,23 & 30 & 1,45 \\
Leche de sapo & - & - & 80 & 3,76 \\
Densidad de población & 1 '620 & 100,00 & $2 ' 130$ & 100,00 \\
\hline
\end{tabular}

*.-Días después de la emergencia. 
Cuadro 4. Efecto de los tratamientos sobre el control de malezas dominantes y toxicidad al cultivo de frijol. Ciclo Otoño-Invierno. 1993-94. CECOT. CIRGOC. INIFAP. SARH.

\begin{tabular}{|c|c|c|c|c|c|c|c|c|}
\hline \multirow[t]{2}{*}{ Tratamiento } & \multicolumn{2}{|c|}{$\begin{array}{l}\text { H. Ancha } \\
\text { \% Control }\end{array}$} & \multicolumn{2}{|c|}{$\begin{array}{l}\text { Zacates } \\
\text { \% Control }\end{array}$} & \multicolumn{2}{|c|}{$\begin{array}{l}\text { Cyperaceas } \\
\% \text { Control }\end{array}$} & \multicolumn{2}{|c|}{$\begin{array}{c}\text { Toxicidad \% } \\
\text { al Cultivo }\end{array}$} \\
\hline & 15 & 30 & 15 & $\mathbf{3 0}$ & 15 & 30 & 15 & $30 *$ \\
\hline $\begin{array}{l}1 \text { Fluazip butil + Fomesafen } \\
0,125+0,25 \mathrm{~kg} \text { i.a./ha }\end{array}$ & 100 & 98 & 100 & 100 & 5 & 5 & 5 & 0 \\
\hline $\begin{array}{l}2 \text { Fluazifop butil + Fomesafen } \\
0,187+0,25 \mathrm{~kg} \text { i.a/ha }\end{array}$ & 100 & 96 & 100 & 100 & 5 & 5 & 5 & 0 \\
\hline $\begin{array}{l}3 \text { Metribuzina } 0,350 \mathrm{~kg} \text { i.a/ha } \\
4 \text { Alaclor + Metribuzina }\end{array}$ & 78 & 76 & 84 & 89 & 5 & 5 & 5 & 5 \\
\hline $\begin{array}{ll} & 1,5+0,21 \mathrm{~kg} \text { i.a/ha } \\
5 & \text { ALACLOR+ Metribuzina }\end{array}$ & 85 & 83 & 95 & 95 & 5 & 5 & 5 & 5 \\
\hline $\begin{array}{l}1,5+0,280 \mathrm{~kg} \text { i.a./ha } \\
6 \text { Pendimetalina + Metribuzina }\end{array}$ & 98 & 93 & 100 & 100 & 5 & 5 & 5 & 5 \\
\hline $\begin{array}{l}0,99+0,21 \mathrm{~kg} \text { i.a./ha } \\
7 \text { Pendimetalina + Metribuzina }\end{array}$ & 94 & 85 & 100 & 100 & 5 & 5 & 5 & 5 \\
\hline $1,32+0,21 \mathrm{~kg}$ i.a./ha & 94 & 89 & 100 & 99 & 5 & 5 & 5 & 5 \\
\hline 8 Imazethapyr $0,078 \mathrm{~kg}$ i.a./ha & 86 & 74 & 100 & 98 & 80 & 80 & 100 & 100 \\
\hline 9 Imazethapyr 0,105 kg i.a./ha & 94 & 90 & 100 & 98 & 85 & 85 & 100 & 100 \\
\hline $\begin{array}{l}10 \text { Imazethapyr 0,131 kg i.a./ha } \\
11 \text { Chlorimuron Ethyl }\end{array}$ & 99 & 83 & 100 & 100 & 95 & 95 & 100 & 100 \\
\hline $\begin{array}{l}\text { 0,0075 kg i.a/ha } \\
12 \text { Chlorimuron Ethyl }\end{array}$ & 100 & 94 & 78 & 53 & 95 & 95 & 100 & 100 \\
\hline $0,0125 \mathrm{~kg}$ i.a/ha & 100 & 98 & 88 & 70 & 97 & 97 & 100 & 100 \\
\hline 13 Bentazon 0,720 kg i.a./ha & 100 & 95 & 89 & 63 & 50 & 50 & 0 & 0 \\
\hline 14 Imazethapyr $0,157 \mathrm{~kg}$ i.a. $/ \mathrm{ha}$ & 98 & 88 & 100 & 100 & 99 & 99 & 100 & 100 \\
\hline 15 Testigo limpio & 100 & 100 & 100 & 100 & 100 & 100 & 0 & 0 \\
\hline 16 Testigo enhierbado & 0 & 0 & 0 & 0 & 0 & 0 & 0 & 0 \\
\hline
\end{tabular}

* Días después de aplicado el producto.

ancha $(76 \%)$ y zacates $(89 \%)$. La mezcla de Alaclor+ Metribuzina en dosis de $1,5+0,210 \mathrm{~kg}$ i.a./ha controló en forma eficiente zacates $(95 \%)$, pero manifestó un control bajo en malezas de hoja ancha $(\leq 85 \%)$, controlando en forma excelente malezas de hoja ancha $(\geq 93 \%)$ y zacates $(100 \%)$, la dosis de $1,5+0,280 \mathrm{~kg}$ i.a./ha. La mezcla de Pendimetalina + Metribuzina a los 15 días después de aplicado el producto (DDA) en dosis de 0,99 $+0,21$ y $1,32+0,21 \mathrm{~kg}$ i.a./ha controlaron eficientemente malezas de hoja ancha (94\%) y zacates $(100 \%)$, pero su efecto de control disminuyó para hoja ancha a los 30 DDA, ocupando porcentajes de control de $85-89 \%$ y siguió mostrando eficiencia para zacates (100\%) confirmando lo indicado por Tamayo (1991) y Esqueda (1992), el Alacolor resultó eficiente para controlar malezas de hoja ancha y angosta en frijol. Ninguno de los herbicidas preemergentes solo ni mezclado controló en forma eficiente coquillo (5\%).

Los herbicidas postemergentes Fluazifop butil + Fomesafén en dosis de $0,125+0,25$ y $0,187+0,25$ $\mathrm{kg}$ i.a./ha controlaron en forma excelente malezas de hoja ancha ( $\geq 96 \%$ ) y zacates ( $100 \%)$, a los 15 y 30 días después de aplicado el producto. La mezcla de estos herbicidas y ejerció ligera toxicidad en el frijol (5\%), en ambas dosis. Estos resultados difieren de los reportados por Morgado y Tafoya (1990) y Ramírez y Tafoya (1990), donde señalan que para tener controles superiores a $80 \%$ es necesario una dosis $0,375 \mathrm{~kg}$ i.a./ha de la mezcla de FLuazifop Butil + Fomesafén o Fomesafén solo. 
Imazethapyr en dosis de $0,078,0,105,0,131 \mathrm{y}$ $0,157 \mathrm{~kg}$ i.a./ha controlaron muy bien zacates por un período de 30 días después de aplicado el producto (98$100 \%$ ). Sin embargo, mostraron controles menos eficientes para hoja ancha, ocupando mejores porcentajes de control las dosis de $0,105,0,131$ y $0,157 \mathrm{~kg}$ i.a./ha con valores arriba de $83 \%$, a los 30 días después de aplicado el producto. Imazethapyr en dosis de $0,078, \quad 0,105, \quad 0,131$ y $\quad 0,157 \mathrm{~kg}$ i.a./ha controló muy bien coquillo ( $\geq 88 \%$ ), siendo mejor las dosis de $0,131 \mathrm{y}$ $0,157 \mathrm{~kg}$ i.a./ha que controlaron excelentemente $(95 \mathrm{y}$ $99 \%$ ) respectivamente.

Es necesario señalar que Imazthapyr en las dosis antes mencionadas causó fitotoxicidad de $100 \%$ en el frijol, expresando la planta como sintomatología una disminución en el crecimiento y clorosis total, no llegando a la muerte de la planta. Los resultados de porcentaje de control concuerdan con los indicados por Ríos y Pérez (1990) y Castro (1993), pero difieren en la toxicidad al cultivo. Mientras que ellos encontraron toxicidad entre $6-10 \%$, nosotros encontramos $100 \%$, que se tradujo en una fuerte reducción del rendimiento. Lo anterior lo atribuimos a las diferencias de los cultivares y a las condiciones climáticas y edáficas existentes.

Chlorimuron Ethyl en dosis de 0,0075 y $0,0125 \mathrm{~kg}$ i.a./ha controló en forma excelente hoja ancha $(\geq 94 \%)$, pero manifestó un control muy ligero sobre zacates (53\%) y fue muy eficiente para controlar coquillo (9597\%) en ambas dosis. Las dos dosis de herbicida causaron toxicidad al cultivo (100\%), mostrando la planta un crecimiento áas lento, arrugamiento y enrrollamiento de hojas y clorosis total que se tradujo posteriormente en la muerte total de la mayoría de las plantas, por lo que este herbicida no es recomendable para aplicarlo en frijol. Lo anterior se debe a que no está autorizado para aplicarlo en frijol, debido a que es un producto no selectivo.

Bentazón en dosis de $0,720 \mathrm{~kg}$ i.a/ha controló eficientemente malezas de hoja ancha $(\geq 95 \%)$ y en forma aceptable coquillo (50\%), pero su efecto fue nulo sobre zacates. Este herbicida no causó efectos tóxicos al frijol. Los resultados confirman lo reportado por Cid y Torres (1987) y Castro (1993).

El análisis de varianza reportó diferencias altamente significativas para las variables altura de planta final (madurez fisiológica), altura final de maleza, plantas cosechadas (miles/ha) y rendimiento de grano en $\mathrm{kg} / \mathrm{ha}$; expresando coeficientes de variación de 24,33, 47,84, 37,23 y $52,13 \%$, respectivamente. Estos coeficientes de variación se consideran altos debido a que los tratamientos donde hubo fitotoxicidad mostraron valores muy bajos en su expresión, lo que ocasionó una amplitud en la varianza estadística.

La mayor altura final de planta fueron los tratamientos con Pendimetalina + Metribuzina en dosis de $1,5+0,210 \mathrm{~kg}$ i.a/ha que fueron estadísticamente iguales a un grupo de doce tratamientos, incluyendo al testigo limpio y al enhierbado; pero diferente a los tratamientos con aplicación de Chlorimuron Ethyl en dosis de 0,0125 y $0,0075 \mathrm{~kg}$ i.a./ha con altura de 10 y 14 $\mathrm{cm}$ respectivamente; lo anterior es debido a fuerte toxicidad causada por este producto.

La menor altura de la maleza al final del ciclo de cultivo fue para los tratamientos con Chlorimuron Ethyl en dosis de 0,0125 y $0,0075 \mathrm{~kg}$ i.a./ha y testigo limpio con 11,18 y $13 \mathrm{~cm}$, respectivamente; siendo estadísticamente diferente a un grupo de 13 tratamientos, incluyendo al testigo enhierbado, lo que da una idea del amplio espectro de control de este herbicida.

El mayor número de plantas cosechadas (miles/ha) fue para el tratamiento con Fluazifop Butil + Fomesafén $0,125+0,25 \mathrm{~kg}$ i.a./ha con $162 \mathrm{mil}$ plantas/ha el cual fue estadísticamente igual a un grupo de 13 tratamientos, pero diferente de Chlorimuron Ethyl en dosis de 0,0125 y $0,0075 \mathrm{~kg}$ i.a./ha donde solo sobrevivieron, al final del ciclo de cultivo, 15 mil plantas/ha, las demás murieron por efectos tóxicos. Lo anterior confirma lo consignado por Cano y López (1993) referente a la alta selectividad de esa mezcla al frijol.

El mejor rendimiento fue para los tratamientos con Fluazifop Butil + Fomesafén 0,125 + 0,25 y 0,187 + 0,25 $\mathrm{kg}$ i.a./ha y Metribuzina con $1,5+0,210 \mathrm{~kg}$ i.a./ha con 1061,999 y $977 \mathrm{~kg} / \mathrm{ha}$, respectivamente, siendo estadísticamente igual por grupo de seis tratamientos, pero diferente del testigo enhierbado e Imazethapyr 0,131, $0,157,0,078,0,105 \mathrm{~kg}$ i.a./ha y Chlorimuron Ethyl en dosis de 0.0125 y $0.0075 \mathrm{~kg}$ i.a./ha que rindieron 290 , $290,212,208,195,44$ y $39 \mathrm{~kg} / \mathrm{ha}$, respectivamente .

Lo anterior indica que los tratamientos con Imazethapyr y Chlorimuron Ethyl rindieron menos que el testigo enhierbado, por lo que se considera que no se pueden utilizar para controlar malezas en frijol ya que disminuyeron los rendimientos en un 82 y $96 \%$ respectivamente en relación al mejor tratamiento, debido a la alta toxicidad causada al frijol (Cuadro 5).

El análisis económico indicó que los beneficios netos para Chlorimuron Ethyl en dosis 0,0075 y 0,0125 
Cuadro 5. Variables medidas en el cultivo de frijol y la maleza para determinar el efecto de los herbicidas. Ciclo OtoñoInvierno 1993-94. CECOT. CIRGOC. INIFAP. SARH.

\begin{tabular}{|c|c|c|c|c|}
\hline \multirow[t]{2}{*}{ Tratamientos } & \multicolumn{2}{|c|}{ Altura final } & \multirow{2}{*}{$\begin{array}{c}\text { Plantas/ha } \\
\text { (Miles) }\end{array}$} & \multirow{2}{*}{$\begin{array}{c}\text { Rendimiento } \\
\text { kg/ha } \\
\text { Tukey } 0,05\end{array}$} \\
\hline & $\begin{array}{c}\text { Frijol } \\
(\mathrm{cm})\end{array}$ & $\begin{array}{c}\text { Maleza } \\
(\mathbf{c m})\end{array}$ & & \\
\hline 1 Fluazifop butil + Fomesafen $0,125+0,25 \mathrm{~kg}$ i.a./ha & $53 \mathrm{~A}$ & $33 \mathrm{BC}$ & $162 \mathrm{~A}$ & $1061 \mathrm{~A}$ \\
\hline 2 Fluazifop butil + Fomesafen $0,187+0,25 \mathrm{~kg}$ i.a. $/ \mathrm{ha}$ & $54 \mathrm{~A}$ & $28 \mathrm{BC}$ & $134 \mathrm{~A}$ & $999 \mathrm{AB}$ \\
\hline 3 Alaclor + Metribuzina 1,5 + 0,210 kg i.a./ha & $56 \mathrm{~A}$ & $67 \mathrm{AB}$ & $147 \mathrm{~A}$ & $977 \mathrm{AB}$ \\
\hline 4 Pendimetalina + Metribuzina $0,99+0,21 \mathrm{~kg}$ i.a/ha & $57 \mathrm{~A}$ & $75 \mathrm{AB}$ & $130 \mathrm{~A}$ & $806 \mathrm{ABC}$ \\
\hline 5 Testigo Limpio & $51 \mathrm{~A}$ & $13 \mathrm{C}$ & $98 \mathrm{AB}$ & $797 \mathrm{ABC}$ \\
\hline 6 Pendimetalina + Metribuzina $1,32+0,21 \mathrm{~kg}$ i.a./ha & $52 \mathrm{~A}$ & $57 \mathrm{ABC}$ & $128 \mathrm{~A}$ & $777 \mathrm{ABC}$ \\
\hline 7 Metribuzina $0,350 \mathrm{~kg}$ i.a./ha & $51 \mathrm{~A}$ & $65 \mathrm{AB}$ & $124 \mathrm{~A}$ & $643 \mathrm{ABCD}$ \\
\hline 8 Alaclor + Metribuzina $1,5+0,280 \mathrm{~kg}$ i.a. $/ \mathrm{ha}$ & $53 \mathrm{~A}$ & $40 \mathrm{ABC}$ & $104 \mathrm{AB}$ & $601 \mathrm{ABCD}$ \\
\hline 9 Bentazon 0,720 kg i.a./ha & $56 \mathrm{~A}$ & $41 \mathrm{ABC}$ & $95 \mathrm{AB}$ & $591 \mathrm{ABCD}$ \\
\hline 10 Testigo Enhierbado & $51 \mathrm{~A}$ & $86 \mathrm{~A}$ & $99 \mathrm{AB}$ & $290 \mathrm{BCD}$ \\
\hline 11 Imazethapyr 0,131 kg i.a./ha & $54 \mathrm{~A}$ & $37 \mathrm{ABC}$ & $115 \mathrm{~A}$ & $290 \mathrm{BCD}$ \\
\hline 12 Imazethapyr 0,157 kg i.a./ha & $54 \mathrm{~A}$ & $24 \mathrm{BC}$ & $101 \mathrm{AB}$ & $212 \mathrm{CD}$ \\
\hline 13 Imazethapyr 0,078 kg i.a./ha & $57 \mathrm{~A}$ & $35 \mathrm{ABC}$ & $70 \mathrm{AB}$ & $208 \mathrm{CD}$ \\
\hline 14 Imazethapyr 0,105 kg i.a./ha & $36 \mathrm{AB}$ & $45 \mathrm{ABC}$ & $71 \mathrm{AB}$ & $195 \mathrm{CD}$ \\
\hline 15 Chlorimuron Ethyl 0,0125 kg i.a./ha & $10 \mathrm{~B}$ & $8 \mathrm{C}$ & $15 \mathrm{~B}$ & $44 \mathrm{D}$ \\
\hline 16 Chlorimuron Ethyl 0,0075 kg i.a./ha & $14 \mathrm{~B}$ & $11 \mathrm{C}$ & $15 \mathrm{C}$ & $39 \mathrm{D}$ \\
\hline Promedio & 47,2 & 41,5 & 100,4 & 532,9 \\
\hline C.V. & 24,3 & 47,8 & 37,2 & 52,1 \\
\hline ANDEVA & $* *$ & $* *$ & $* *$ & $* *$ \\
\hline
\end{tabular}

$\mathrm{kg}$ i.a/ha fueron negativos y para el caso de Imazethapyr, ligeramente positivos. Los tratamientos con mejor tasa marginal de retorno fueron Metribuzina $0.350 \mathrm{~kg}$ i.a. $/$ ha Alaclor + Metribuzina $1,5+0,290 \mathrm{~kg}$ i.a./ha y Fluazifop Butil + Fomesafén 0,125 +0,25 kg i.a./ ha con un TMR de 444,1655 y $36 \%$ y relación beneficio costo de 8,$9 ; 10,6$ y $6,3 \%$ respectivamente (Cuadro 6).
El mejor tratamiento desde el punto de vista económico fue Alaclor + Metribuzina en dosis de 1,5 + 0,210 kg i.a/ha; aunque Fluazifop Butil+ Fomesafén resultó excelente para controlar malezas, el análisis económico lo descarta por ser muy elevada la inversión; sin embargo, los tratamientos se pueden usar cuando el control así lo amerite, porque los tres tratamientos resultaron eficientes en control y eco-

Cuadro 6. Análisis de la tasa marginal de retorno de los mejores tratamientos de herbicidas en frijol. Ciclo OtoñoInvierno. 1993-1994. CECOT. CIRGOC. INIFAP. SARH.

\begin{tabular}{|c|c|c|c|c|c|c|}
\hline Tratamientos & $\begin{array}{c}\text { Rend. } \\
\text { kg/ha } \\
\text { (N \$) }\end{array}$ & $\begin{array}{c}\text { Benef. } \\
\text { Bruto } \\
\text { (N \$) }\end{array}$ & $\begin{array}{c}\text { Costos } \\
\text { Variab. } \\
\text { (N \$) }\end{array}$ & $\begin{array}{c}\text { Benef. } \\
\text { Neto }\end{array}$ & $\mathbf{R B C}$ & $\begin{array}{c}\text { TMR } \\
\%\end{array}$ \\
\hline 16 Testigo Enhierbado & 290 & 609 & 0 & 609 & - & - \\
\hline 3 Metribuzina 0,350 kg i.a./ha & 643 & 1350 & 136 & 1214 & 8.9 & 444 \\
\hline 4 Alaclor + Metribuzina 1,5 + 0,21 kg i.a/ha & 977 & 2052 & 176 & 1876 & 10.6 & 1655 \\
\hline 1 Fluazifop Butil + Fomesafen $0,125+0,25 \mathrm{~kg}$ i.a. $/ \mathrm{ha}$ & 1061 & 2228 & 305 & 1923 & 6.3 & 36 \\
\hline
\end{tabular}

$\mathrm{TMR}=$ Tasa marginal de retorno.

$\mathrm{RBC}=$ Relación Beneficio-Costo . 
nómicos. Lo anterior nos permite ofrecer tecnología para distintas alternativas de infestación de maleza en el centro de Veracruz y regiones similares (Cuadro 6).

\section{CONCLUSIONES}

1. Las malezas dominantes en el sitio experimental fueron flor amarilla (Aldama dentata) y zacate pitillo (Ixoporus unisetus).

2. Los herbicidas preemergentes Metribuzina 0,350 kg i.a./ha y Alaclor + Metribuzina 1,5+0,210 kg.i.a/ ha fueron eficientes para controlar malezas de hoja ancha y zacates y los más indicados desde el punto de vista óptimo económico.

3. La mezcla de los herbicidas postemergentes Fluazifop Butil+Fomesafén en dosis 0,125 + 0,25 $\mathrm{kg}$ i.a./ha controló excelente zacates y hoja ancha (100\%), siendo el más indicado de los postemergentes en forma óptima económica cuando las malezas dominantes sean zacates y hoja ancha.

4. El mejor tratamiento óptimo-económico fue Alaclor + Metribuzina 1,5+0,210 kg i.a/ha.

5. Imazethapyr y Chlorimuron Ethyl son altamente tóxicos para el frijol en el trópico Veracruzano.

\section{LITERATURA CITADA}

BOLAÑOS, E.A.; HERNANDEZ, R.B.R.; LAZAINZ, B.A.A. 1993. Control químico de maleza en frijol ejotero (Phaseolus vulgaris L) Var. Black Valentine. In: IV Congreso Nacional de la Ciencia de la Maleza Puerto Vallarta, Jalisco. México. pp.58.

CANO, R.O.; LÓPEZ, S.E. 1993. Estudio de factibilidad para la producción artesanal de semilla de frijol en la región de la Mixtequilla en Veracruz. México. Documento Interno sin publicar.

CASTRO, M.E. 1993. Control químico de cadillo Xanthium stromarium L. en frijol en la región lagunera. In: Memoria XIV Congreso Nacional de la Ciencia en la Maleza, Puerto Vallarta, Jalisco. México. pp. 56.
CID, J.J.G.; TORRES, G.A. 1987. Evaluación de dosis de los herbicidas fomesafén y bentazón para el control de malezas en el cultivo de frijol (Phaseolus vulgaris). En la región de Yanga, Ver. In: Memorias del VIII Congreso Nacional de la Ciencia de la Maleza. San Luis Potosí, México. pp. 297-302.

ESQUEDA, E.V.; LOPEZ,S.E.; CANO R.O. 1993. Efecto de la dosis y época de aplicación de fomesafén en la maleza de hoja ancha del frijol de riego. In: Resultados de investigación en el cultivo de frijol 1992. Proyecto CONACYT-CARNATION-NESTLE-INIFAP-CECOTCIRGOC-INIFAP-SARH. Informe Técnico División Agrícola, Veracruz, Ver. México. p. 60-66.

ESQUEDA, E.V.A.; LOPEZ, S.E. 1992. Evaluación de herbicidas en el cultivo de frijol de humedad residual. In: Informe Técnico. Resultados de investigación sobre frijol 1991. Programa Leguminosas Comestibles. Proyecto Colaborativo. INIFAP-CONACYT-CARNATIONGOB. VER.CECOT-CIRGOC-INIFAP-SARH. Veracruz, Ver. México. pp. 51-57.

ESQUEDA, E.V.A.; LOPEZ, S.E. ; CANO, R.O. 1992. Dosis y época de aplicación de fomesafén en maleza de hoja ancha en frijol. In: Memoria de XIII Congreso de la Ciencia de la Maleza, Chapingo, Estado de México. p. 53.

RAMÍREZ, F. M.; TOFOYA, R.J.A. 1990. Control de la maleza en frijol (Phaseolus vulgaris $\mathrm{L}$ ). Sembrado con dos distribuciones y en labranza de conservación. In: Memoria del XI Congreso Nacional de la Ciencia de la Maleza. Irapuato Gto. México. pp. 84.

RIOS, T.A.; ROJAS, G.M. 1987. Efectos fitotóxicos del fomesafén en frijol (Phaseolus vulgaris L) y su acción en anotomía del tallo. En VIII Congreso Nacional de la Ciencia de la Maleza. San Luis Potosí, S.L.P. México. pp. 87-87.

RIOS, T.A.; PEREZ, M.L.A. 1990. Control químico postemergente de maleza en frijol. In: XI Congreso Nacional de Ciencia de la Maleza. Irapuato, Guanajuato, Méx. pp. 47.

MORGADO, H.G.; TAFOYA, R.A. 1990. Control de la maleza en frijol (Phaseolus vulgaris L). Sembrado con dos distribuciones. En XI Congreso Nacional de la Ciencia de la Maleza. Irapuato, Gto. México. pp. 46.

TAMAYO, E,L.M. 1991. La investigación sobre maleza y su manejo integrado. En: Series técnicas de ASOMECIMA. Número (1) volumen II,. México, D.F. pp. 29-39. 